\title{
Anthropometric Survey of the Spanish Female Population Aimed at the Apparel Industry
}

\author{
Sandra ALEMANYª , Juan Carlos GONZÁLEZ ${ }^{\mathrm{a}}$, Beatriz NÁCHER ${ }^{\mathrm{a}}$, Carol SORIANO ${ }^{\mathrm{a}}$, \\ Carlos ARNÁIZ $Z^{\mathrm{b}}$, Ángeles HERAS ${ }^{\mathrm{b}}$ \\ anstituto de Biomecánica de Valencia, Valencia, Spain. \\ ${ }^{b}$ Instituto Nacional de Consumo, Madrid, Spain
}

\begin{abstract}
Sizing systems used by the apparel industry are not always accurately optimized to fit the majority of a specific population. One of the main problems to develop new patterns and grade to all sizes is the lack of updated anthropometrical data.
\end{abstract}

Additionally, 'vanity sizing', where companies often adjust the measurement specifications for each size based on a sale strategy designed to make consumers, especially women, feel better about fitting into smaller sizes [1] [2] [3]. This and the lack of international common standards to size clothing contribute to difficult customers to find the correct size

In this context, the Instituto Nacional de Consumo (INC) of the Spanish Ministry of Health, supported by the main Spanish companies of the apparel industry, proposed the Biomechanical Institute of Valencia to carry out an anthropometric study to characterize the morphology of the female population in Spain.

The target sample of the national campaign was 10.141 women grouped into 10 age ranges from 12 to 70 years. The measurements were made in 61 different locations, representing the seven Spanish NUT areas (statistical territorial units defined by the European Community). Using a 3D body scanner (Vitus Smart from Vitronic), two scanning postures were registered for each subject. Weight measure and a socio-demographic survey including questions about apparel purchase habits were included in the measurement protocol. The sample was randomly selected based on the census of the population of each town and city, considering age as the control variable. The management of contacts and appointments of the selected users was guided by the Ministry of Health, and the participation was voluntary.

The objective of this paper is to present the methodology of the national measurement campaign and the main results obtained from the preliminary analysis: anthropometric measures, morphotypes and their evolution with the age, which could be applied to improve the apparel fitting.

Keywords: Anthropometry, 3D scanning, apparel, fitting.

\section{Introduction}

Clothing fit is the main objective in the garment development process to ensure user comfort and appearance. It is a complex aspect influenced on one side by the anthropometry and body dimensions of the customer and, in the other, by the appearance and social trends [4].

'Vanity sizing', where companies often adjust the measurement specifications for each size, is a sale strategy designed to make consumers, specially women feel better about fitting into smaller sizes [1] [2] [3], and therefore prompting them to buy more. This practice, allowed by the lack of common standards to size and labeled the cloth, promotes an unhealthy image of beauty, and could even contribute to create eating disorder psychopathologies [5]

Anthropometric studies carried out since the moment support this fact: studies developed in the UK (Size UK, 2001-2002, [6]) and Germany [7] show a $60 \%$ and $50 \%$ of customers with difficulty finding clothes that fit properly. In the same way, an anthropometric study done in USA (Size USA) to update the sizing ASTM standards also concluded that a $54 \%$ of the population was not satisfied with the fitting of the RTW cloth [8].

* sandra.alemany@ibv.upv.es; http://www.ibv.org/en.html 
On the other hand, the industrialization process of new garments development has a significant influence in the fitting properties of a new collection, specifically, regarding ready to wear (RTW) cloth, being one of the main problems the deviation of the industrial parameters from the anthropometry of the target population.

In this sense, the development of RTW clothing requires an estimation of body measures of the target population. However, the common practice of most apparel manufacturers is to create and adjust their own size charts by trial and error by means of small customer surveys, mainly models representing the basic size, analysis of sales and returned merchandise reports [9]. The growing relocation of the pattern and production activities and the poor level of application of sizing standards also produce that one of the main clothing complaints is the lack of fitting.

Although the integration of 2D CAD packages in the new pattern development process is widely extended, and has reduced significantly the timescale to industrialize a new design, the design of the pattern in the basic size remains a bottleneck. The lack of fitting criteria and anthropometric data of the target population to guide the designer in this process requires always the construction of prototypes and a final fitting test with real models with a consequent increment of cost and time to market.

Since fitting RTW cloth is a problem for both the customer and the apparel industry [1], national administrations and industrial groups of the clothing sector have been fostering, during the last years, national anthropometric surveys in different countries: EEUU, UK, France, Australia, Spain and Germany among others. Additionally, from the technological point of view new 3D body scanning techniques constitute a step forward in the way of conducting and analyzing anthropometric data and contribute to promote new anthropometric surveys. As a result, broad anthropometric databases are available and constitute a high valued data to improve garment fitting adapted to the body shape of the population.

CAESAR was the first anthropometric survey to provide 3D human models [10]. The project that gathers more than 13.000 scan data was a survey of the civilian population in three countries: USA, the Netherlands and Italy. Lessons learned from this project were used to plan most of the subsequent studies. The experimental design to define the sample of the population, the process to recruit the subjects, the scanning technology and the measurement protocol were the main keys of any 3D anthropometric survey defined to cover the specific objectives proposed in each national campaign.

This paper presents the methodology developed to perform the anthropometric study of the female population in Spain. Data was collected in 61 different locations over a four months period using six scanners working at the same time. A previous pilot study was carried out to set up the methodology with the objective to analyze subject recruitment and the level of participation, the accuracy of the process and optimize the duration of the session and the logistic aspects.

The anthropometric study of the female population in Spain was promoted by the Ministry of Health supported by the main Spanish companies of the garment industry and developed by with the Institute of Biomechanics of Valencia and researchers from the statistical and nutrition areas. The aim of the anthropometric survey was to generate anthropometric data from the female population addressed to the clothing industry and to promote a healthy image of beauty.

\section{Experimental design and subject recruitment}

Three control variables were used to define the experimental design: age, geographic area and country size. The age was classified in ten groups from 12 to 70 years old (table 1). Spanish territory was divided into seven areas according to the NUTS (nomenclature of territorial units for statistics) European classification and three levels were defined for the country size depending on the number of inhabitants: $<10.000,10.000-500.000,>500.000$.

First, the calculation of the sample size was done based on the weight variable to obtain a level of error under $\pm 1 \%$ for each group of age with a $95 \%$ level of confidence. For the whole population the expected error was lower than $0.25 \%$. With these requirements, the final target sample size was 10.415 females. Second, 61 geographical points of measure (figure 1) were selected randomly based on the three level classification. Finally, the distribution of the sample was done proportional to the number of population and the participants in each location were randomly selected from the official Postcode Address File. 
An invitation to participate in the study was sent from the Ministry of Health to the selected women including a free phone number to arrange the measure session. An SMS was sent the day before the appointment to remind the measurement appointment.

Only for women under 18 years old, it was required for the data collection a Consent Agreement signed by their guardian.

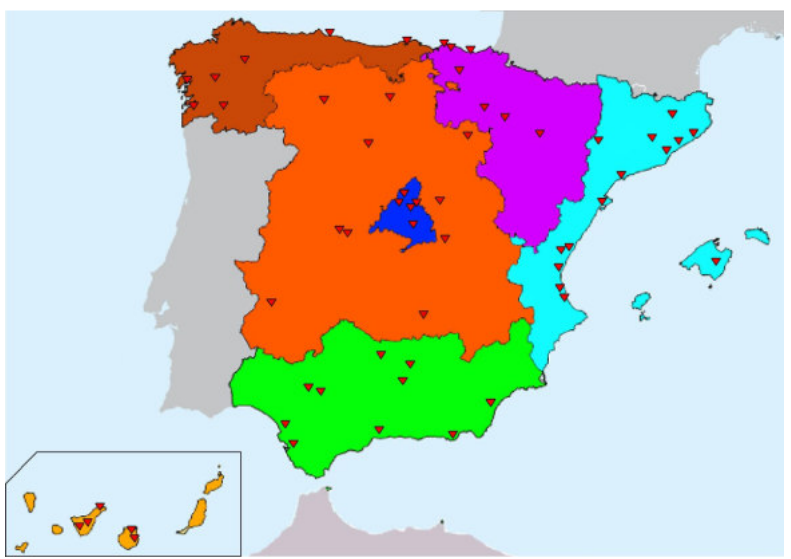

Table 1. Definition of groups of age
\begin{tabular}{|c|c|}
\hline $\begin{array}{c}\text { Group of } \\
\text { age }\end{array}$ & Range \\
\hline 1 & $12-13$ \\
\hline 2 & $14-15$ \\
\hline 3 & $16-17$ \\
\hline 4 & $18-19$ \\
\hline 5 & $20-24$ \\
\hline 6 & $25-29$ \\
\hline 7 & $30-39$ \\
\hline 8 & $40-49$ \\
\hline 9 & $50-59$ \\
\hline 10 & $60-70$ \\
\hline
\end{tabular}

Fig. 1. Distribution of measurement points

As the percentage of participation was previously unknown, the process of random sample selection was repeated until eight times. Chained recruitment cycles were done over-sizing the call for volunteers in order to cover the whole sample size for each group of age anticipating a percentage of letters without answer.

To promote the participation of subjects, a communication campaign was defined. At the beginning of the study, the Instituto Nacional de Consumo lunched the project with a press conference including all the main national media. During the study, local promotion by means of radio, newspaper, television and press conferences where done in every town to keep a high level of participation.

Additionally, a wide timetable schedule of the data collection sessions was defined, including weekends to facilitate the participation of women out of their job activity.

\section{Data collection}

The The Vitus Smart 3D body scanner from Human Solutions [11] was used in the Spanish survey. It is a non-intrusive laser system formed by four columns allocating the optic system, which moves from the head to the feet in ten seconds performing a sweep of the body.

One of the critical aspects of this study was the great number of geographical points of measure, which required many scanner displacements, assemblies and dismantles with consequent fails, broken components and calibrations. The result of the experimental design required the transportation of each of the six scanners every week. In order to minimize risks, all the manipulation, displacements and installations of the scanners where done by an instructed technician limiting the use of courier companies only to ship the scanner to the Canarias islands. Three teams of two people each one were in charge of this process based on a protocol including a strict calibration and a checking process at the end of each new installation. Finally, the evaluation of the data concluded with confirmation of the equipment manager, which was required to start with the data collection. An additional backup scanner was available at IBV to exchange any piece if required.

The scanning system allows obtaining until 130 anthropometric measures automatically based mainly in characteristic points obtained geometrically. However, the accuracy to find some anatomical points, relevant for anthropometric measures used in garment patterning, is not enough, mainly because these points are not reflected geometrically in the body surface. Thus, seven anatomical points (right and left acromions, right and left bottom part of the tenth ribs, right and left liac spines and the seventh cervical) were manually detected by an instructed specialist and semi-sphere makers with anti-allergic sticks were placed (figure3). 
Standard white garment, a swimming hut, a top and a short, was design and scaled in 5 sizes, in order to harmonize the measurements (figure 2). The design of the garment was base on the standard ISO 20685 and an estimation of the size distribution was done from the pilot study for logistic management issues.

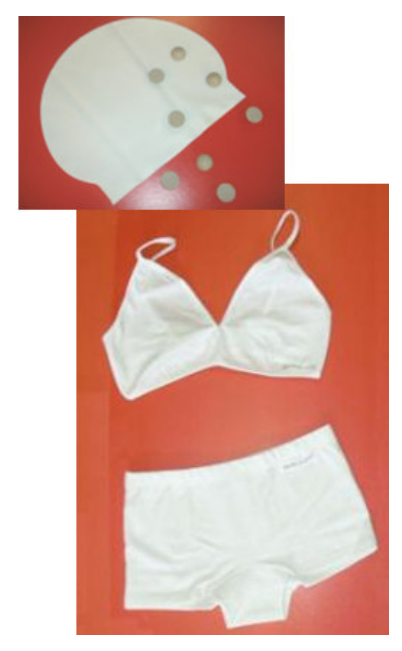

Fig. 2: Standard cloth for the measurement process
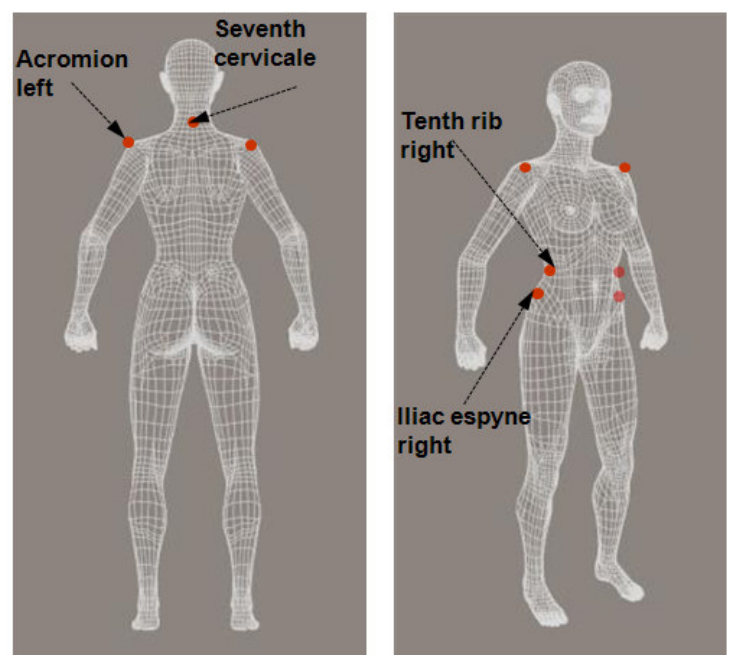

Fig 3.Anatomical markers

Subjects' weight was gathered using the weight scale SECA integrated in the scanner pedestal. To conclude, a questionnaire about socio-demographic data, common garment size and healthy habits like level of sport practice and diet were also registered using a PDA.

Both, recruitment and measurement process satisfy the Data Protection regulation in Spain. Therefore, the measurements were anonymous and identified with a code used to integrate data coming from the questionnaire and the 3D scanner.

Two women carried out the measurement session. One, with a health background, was in charge of the subject assistance, manual measures and markers placement. The other technician with a technologic background monitored the scanner, perform the daily calibration and check the correct operation of the scanner. This measurement team allowed to overlap the measurement of two subjects at the same time.

The measurement process for each session was the following:

- The subject was welcomed and the measurement process explained.

- Two manual measures (bust and hip girth) were taken to select the size of the standard top and short used in the measurement process.

- Into the dressing room, the technician took manually the skin folds measures: suprailiac, tricipital and scapular. Each measure was repeated three times.

- After that, the seven anatomical points were manually detected and semispherical makers with anti-allergic sticks were placed.

- Then the subject entered the scanning cabinet and three measures were taken in two postures: standard and standing. In addition, the sitting posture was scanned only for the $25 \%$ of the population randomly selected (figure).

- Finally, once dressed, women filled on the questionnaire driven by the technician.

Although the complete measurement session only took between 15 and 20 minutes, the bottleneck to speed up the data collection was the subject recruitment process, which resulted on a mean number of subjects measured per day of 20 . 


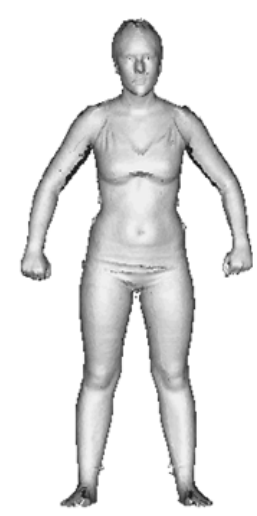

STANDARD

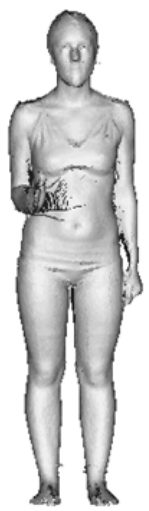

STANDING

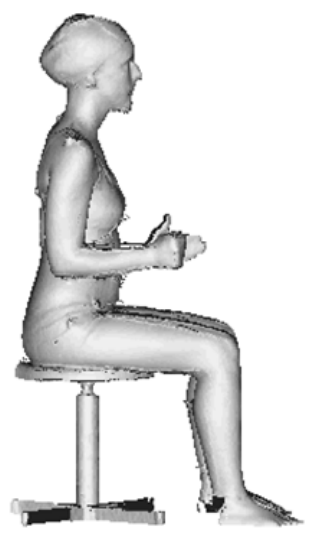

SITTING

Fig. 4: Scanning postures.

\section{Data processing}

Anthroscan software [12] was used to extract 95 anthropometric measures from each 3D mesh. Despite the software performs the data extraction automatically, there were some measures, presenting high sensibility to the scanning posture, and also areas with fat concentration, which require a manual extraction. For example, the armpit points were usually automatically detected under the real points in subjects with overweight so they had to be manually placed.

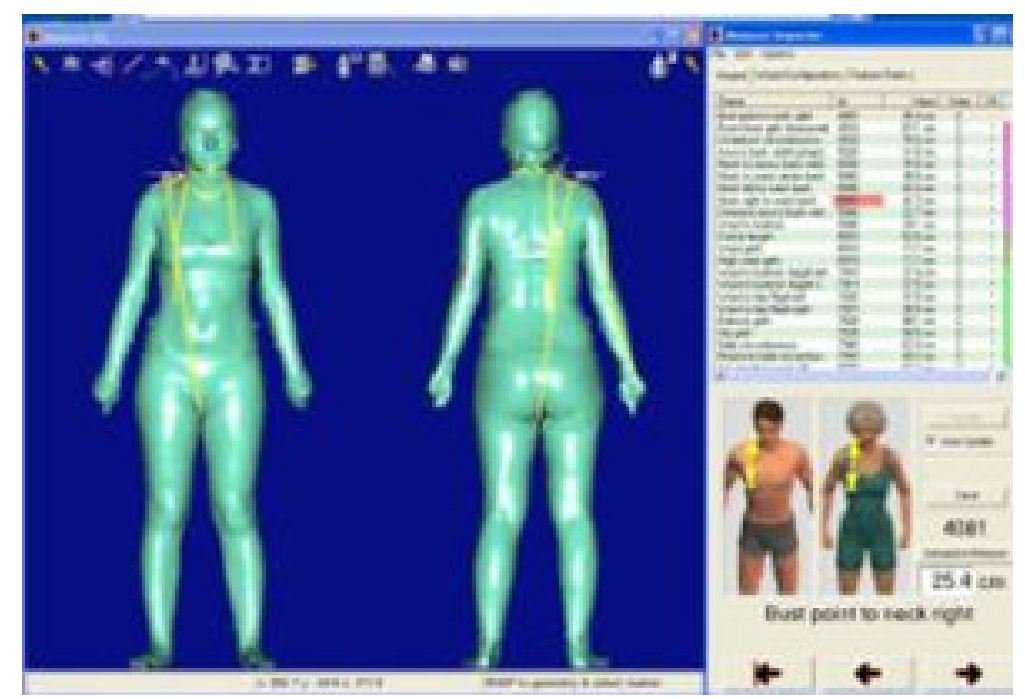

Fig. 5: Automatic measurements

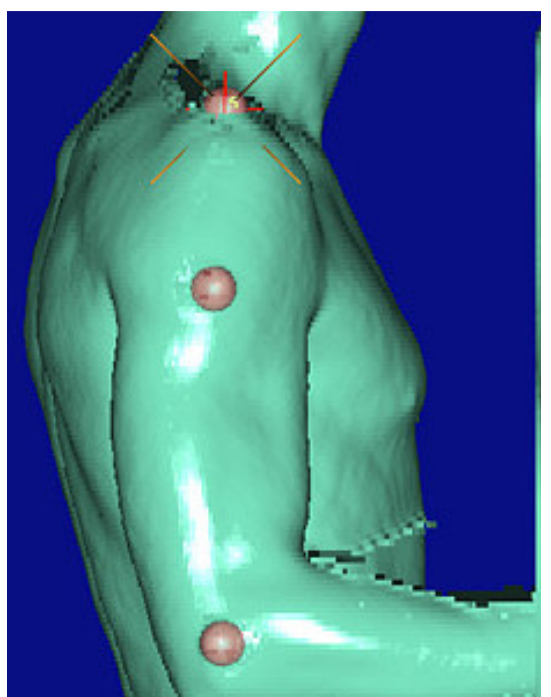

Fig. 6: Semi-automatic detection of markers

This semi-automatic methodology combining automatic measures (figure 5) with a manual review (figure 6) was applied during the measuring extraction phase to ensure the measurement quality. In parallel with the measurement process, three persons were revising each $3 \mathrm{D}$ scan an extracting the anthropometric measures. This configuration allowed detecting measurement problems in early stages of the process.

Specific software was developed to integrate the complete the anthropometric database linking the anthropometric measures with the 3D model and the questionnaire results through a user code. Filtering and exportable utilities were added to assist the database configuration ready to analyze with statistical and mathematical tools. 


\section{Preliminary results}

In general, the level of participation was very high. However, the tight range of age in the younger groups entailed a difficulty for the recruitment process for these groups so they registered the lower level of participation (figure 7).

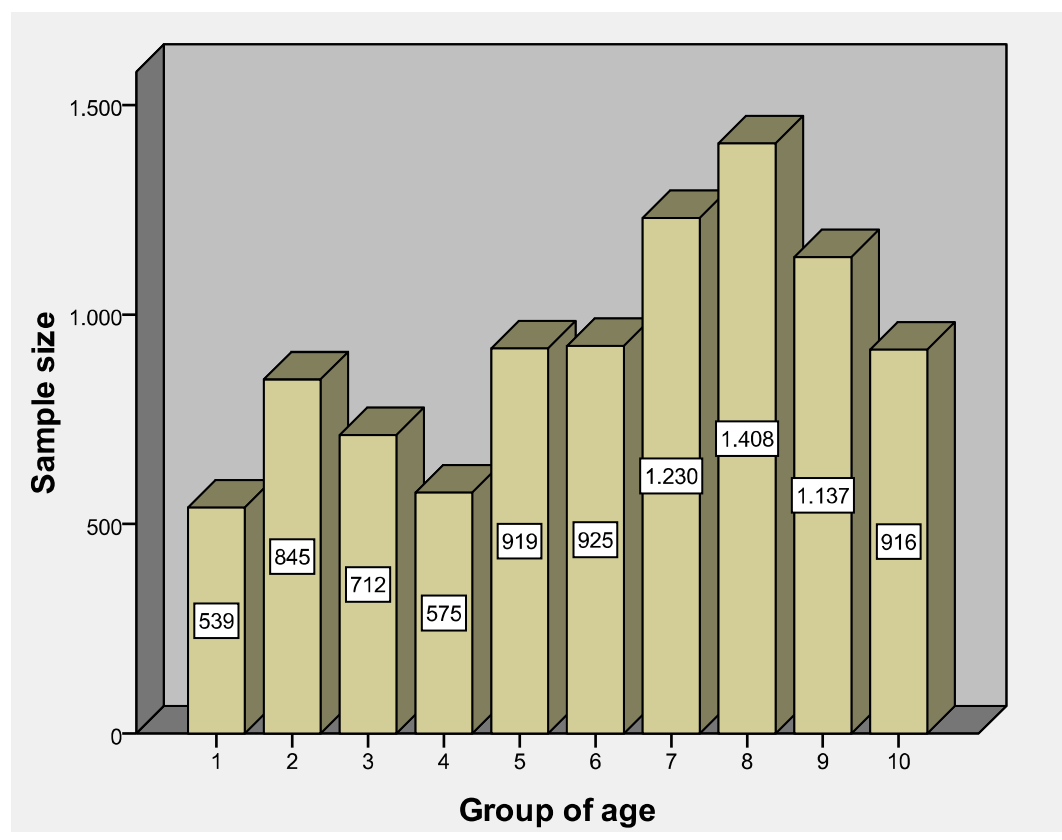

Fig. 7: Simple size for each group of age.

A $40 \%$ of the population has problems to find their appropriate size. This result agrees with previous studies performed in other countries. Regarding the causes of the fitting problems, the $43 \%$ answer a lack of larger sizes. The figure 8 shows a correlation between the body mass index and the problems to find cloth.

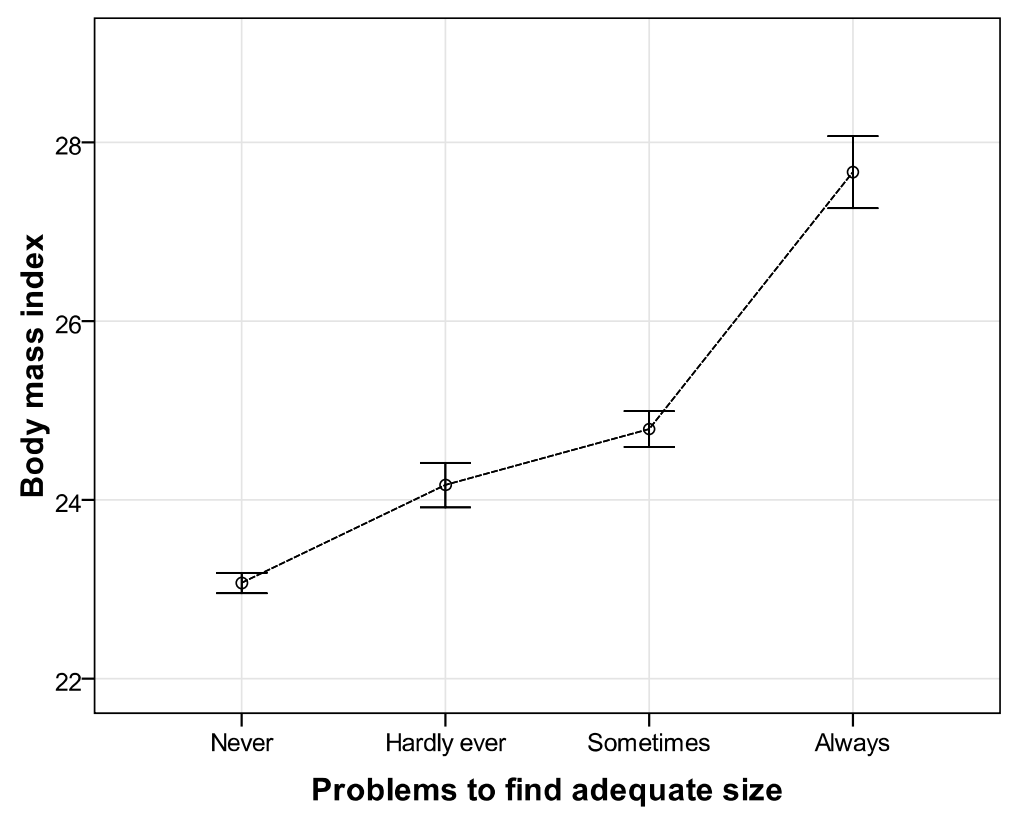

Fig.8: Problems to find adequate size related to body mass index. 
The design of experiments performed in the present study allowed the analysis of anthropometry variability across the age with high resolution. This is very valuable information from anthropologic point of view but also, to define accurately products for small groups based on age.

As an example, the evolution of the stature with the age (figure 9) shows the growing process in the first stage while, in the last stage, it is showed a stature reduction. The shrinkage of the population is due to a combination of a 'secular trend' explained by the development of the social and economic conditions until 40s and the aging process after around the age of 50.

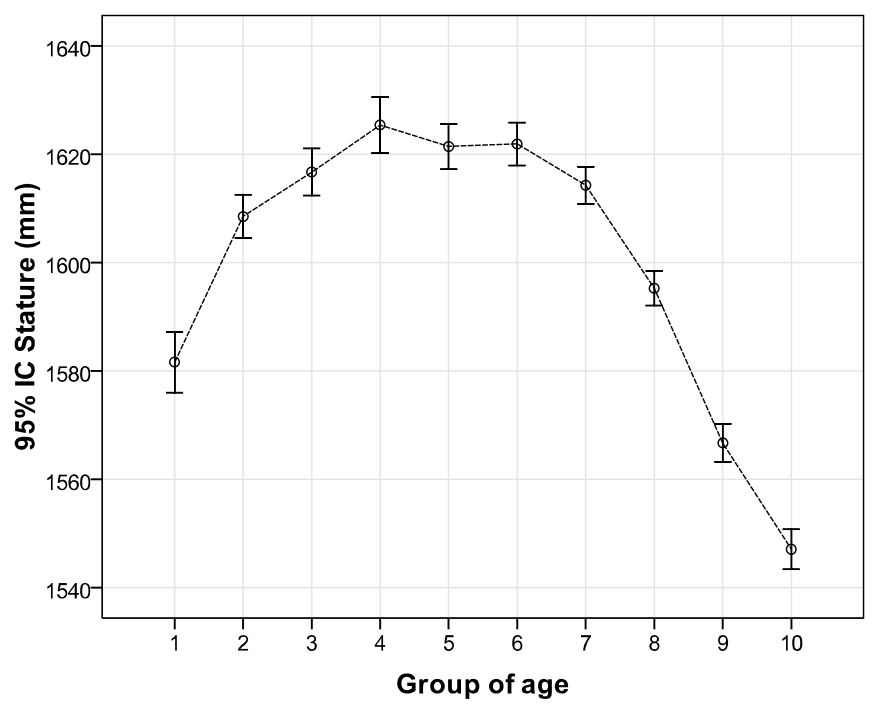

Fig. 9: Effects of group of age on stature.

The weight (figure 10) evolution is completely different and shows an increasing trend through the age. Combined with the shrinkage process, the result is a significant increment of the body mass index with the age.

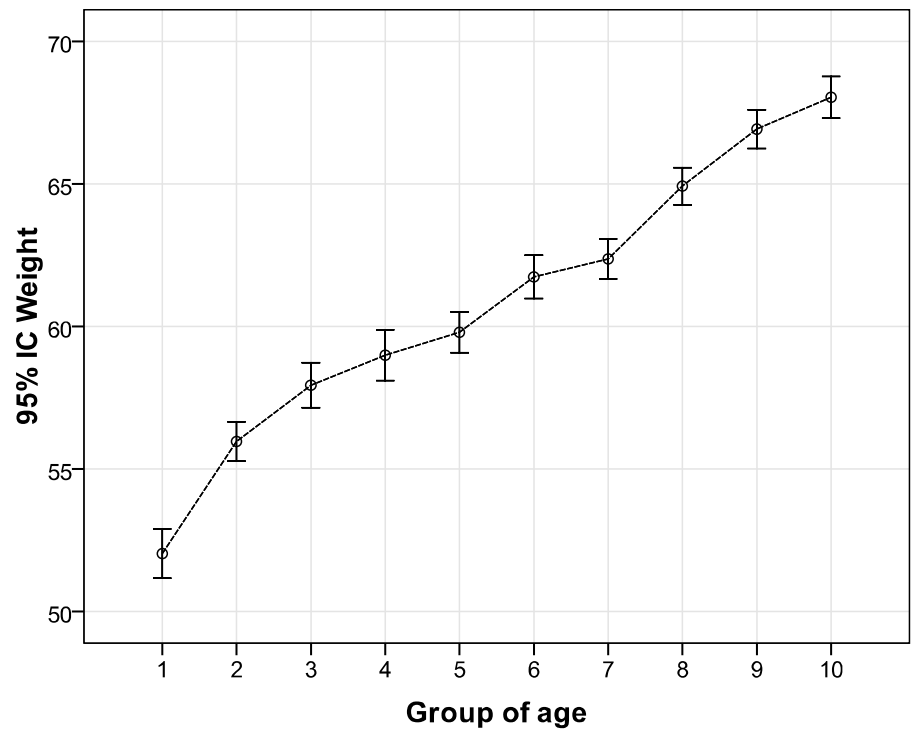

Fig. 10: Effects of group of age on weight $(\mathrm{kg})$.

From the perspective of the clothing industry to improve the garment fitting, the results provided by this study are essential to optimize design and sizing for specific target groups. The progression of the waist and bust circumference with the age (figures 11 and 12), primary measures to define garment size, suggests an optimized segmentation of sizes methods based on anthropometric measures. 


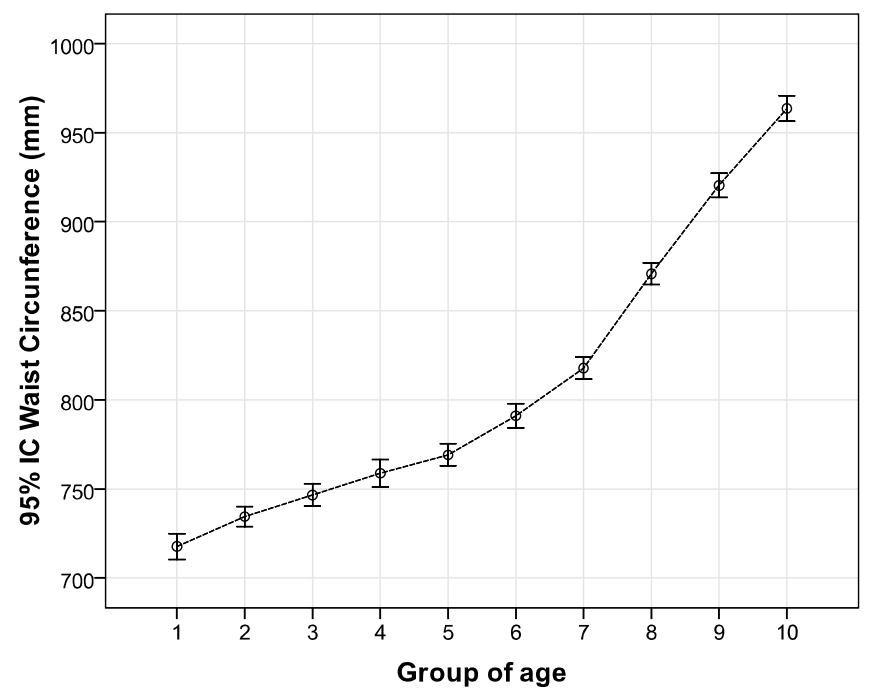

Fig. 11: Effects of group of age on waist circumference.

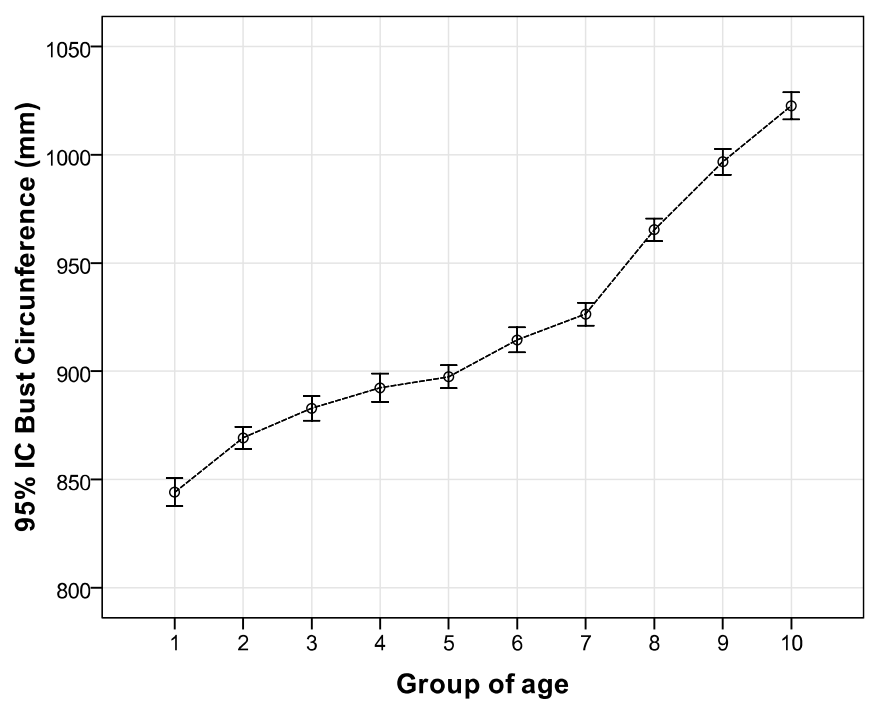

Fig. 12: Effects of group of age on bust circumference.

The analysis of the three main women morphotypes based on the bust, waist and hip circumference ratios (figure 13), revels a change in the body shape with the age produced by a greater increase on the hip circumference.

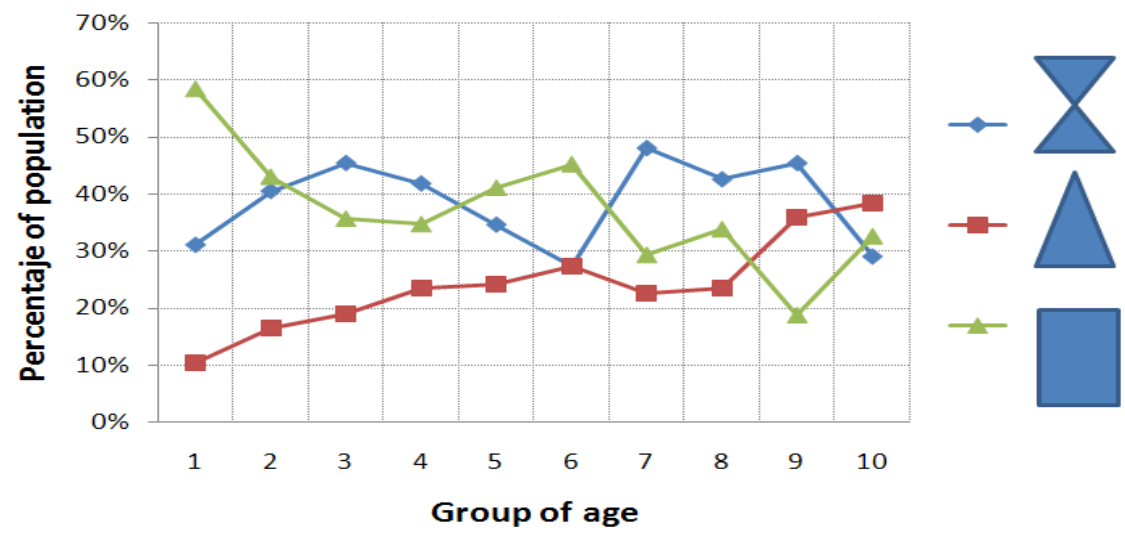

Fig. 13: Effects of group of age on morphotype. 


\section{Conclusions}

Updated anthropometry data of the target population constitutes valuable information to improve garment fitting, optimize the sizing system and reduce the design process cycle. Nevertheless, clothing fit is a complex issue combining aspects as 3D body shape but also textile mechanical properties, clothing drape and fashion trends. Additional research work must be carried out to rationalize the problems of fit and explain its complexities using a logical approach and understanding [4].

However, to reach the final customer, the sizing standardization is one of the main needs. Nowadays European research situation in anthropometrics, with similar national surveys were/being done in many countries across the European Union, allows to think that the improvement of clothing fitting by means of a common European sizing designation standard could be tackled now. The European Committee for Standardization (CEN/TC 248/ WG 10) proposed a new sizing system based on key body dimensions. An integration effort of the anthropometrical data generated in different European regions is required in order to complete the development of this standard.

An additional challenge of these surveys is the exploitation of the 3D human models. New technologies developed to generate garment patterns from 3D models and the integration of avatars in virtual catwalks and virtual try-on services require flexible, quick and easy to use tools to manage these databases. A combination of virtual technology and accurate data coming from the analysis of 3D human model databases is one of the key points to enhance two of the emerging business models in the clothing industry: internet sales and clothing customization.

\section{References}

1. Pisut, G. y Connell, L.J. (2007): "Fit preferences of female consumers in the USA". Journal of Fashion Marketing and Management Vol. 11 No. 3, 2007, pp. 366-379.

2. La Bat K L and DeLong M R, (1990): 'Body cathexis and satisfaction with fit if apparel'. Clothing and Textiles Research Journal January, 8(2) 43-48.

3. Phoebe R. Apeagyei, Rose Otieno and David Tyler (2007): "Ethical practice and methodological considerations in researching body cathexis for fashion products". Journal of Fashion Marketing and Management. Vol.11; no3; pp. 1361-2026.

4. Fan. J., Yu, W. and Hunter, L., (2004). Clothing appearance and fit: Science and technology. Woodhead Publishing Limited.

5. Joanna K. Vallance (2006): "The relationship between eating disorder psychopathology and quality of life within a nonclinical Sample", Thesis submitted in partial fulfilment of the requirements for the Degree of Master of Arts in Psychology. University of Canterbury.

6. Fashion Technology, Size and Fit Solutions: www.sizemic.eu/products-and-services/46-size-survey-data

7. Matrix GmbH (1999): "Zitex-Studie: Transfer-Workshop Results of the Feasibility Study for Industrial Made-to-Measure Apparel".

8. Smith D.C. (2007): "Process, fit and appearance analysis of three-dimensional to two-dimensional automatic pattern unwrapping technology". Thesis submitted to the Graduate Faculty of North Carolina State University.

9. Sizing in clothing (libro)

10. Robinette K. and Daanen H. (year): "Lessons learned from CAESAR: A 3-D anthropometryc survey".

11. Body scanning system "Vitus Smart" (accessed 2010): www.human-solutions.com/apparel/technology_scanning_en.php

12. 3D data processing software "Anthroscan" (accessed 2010): http://www.human-solutions.com/apparel/products_anthroscan_en.php 